\title{
Growth features and nitrogen aggregation properties of microdiamonds derived from kimberlitic diatremes
}

\author{
Trautman, R.L. ${ }^{1}$, Griffin, B.J. ${ }^{2}$ and Bulanova, G.P. ${ }^{3}$ \\ 1. Department of Geology \& Geophysics, University of Western Australia, Nedlands 6907, Australia \\ 2. Centre for Microscopy \& Microanalysis, University of Western Australia, Nedlands 6907, Australia \\ 3. TsNIGRI, 129B Varshavsky Shosse, Moscow 113545, Russia (galina_bulanova@hotmail.com)
}

\section{Introduction}

Establishing a universal origin for microdiamonds is greatly complicated by the range of physical and chemical properties seen in large populations of these stones, nevertheless, many suites share similar trends. These trends can be used to constrain the circumstances surrounding the genesis of microdiamonds. With few exceptions, microdiamonds extracted from kimberlite diatremes retain a high proportion of octahedral growth forms that are commonly accompanied by stones of cubic, or more rarely, cubo-octahedral morphology. Coexisting in this size fraction are resorbed or rounded species which customarily share some of the characteristics preserved by their primary counterparts. However, it is the survival of the octahedral form within microdiamond suite that has been the subject of most interest. A number of genetic models have been advocated for their origin (Haggerty, 1986; Pattison and Levinson, 1995). These include evolution in the mantle environment with or without the influence of mantle-derived fluids and development in the kimberlite melt itself, but as noted by Pattison and Levinson (1995), very few studies to date are supported by data that can adequately resolve this debate.

In order to evaluate the above hypotheses over 1600 microdiamonds extracted from 12 geographically diverse kimberlite pipes (covering Australia, Russia, Brazil, Finland and South Africa) have been investigated for their morphological (both external and internal) and nitrogen aggregation characteristics. The nitrogen contents and aggregation states for whole microdiamonds, all of which measure less than $0.5 \mathrm{~mm}$, were determined by Fourier Transform Infra-red (FT-IR) spectroscopy using the facilities and procedure outlined in Trautman et al (1997). The nitrogen characteristics of many of these microdiamonds were reported in an earlier work (Trautman et al, 1997), but the recognition that diamond may have complex zonation on the basis of its nitrogen distribution ( provided the impetus for studies based on their cathodoluminescence properties. Whereas external morphology gives some indication of the final stages of diamond growth (or dissolution) prior to surface emplacement, investigation of a diamond's internal structure provides information on much of its growth history. 141 polished microdiamond sections have been examined by cathodoluminescence under a constant set of conditions; $15 \mathrm{kV}$ accelerating voltage, $25 \mathrm{~mm}$ working distance and $3 \mathrm{nA}$ beam current, using a JEOL 6400 scanning electron microscope coupled to a cathodoluminescence detector . Preparation of internal sections for cathodoluminescence work involved embedding the microdiamond samples within epoxy resin prior to polishing to a central level using a low-speed diamond lap.

\section{Cathodoluminescence data}

Cathodoluminescence imaging has revealed not only the existence of internal zonation among kimberlite-derived microdiamonds, but a large degree of internal variability that provides strong evidence for complex, multistage growth histories. In most cases the evolutionary sequence appears unrelated to the external morphology of the crystal and instead follows one of three general trends noted by Bulanova and Milledge (1995) to occur in macrodiamonds; 1) homogeneity, which may involve subtle internal variations in cathodoluminescence intensity, 2) simple octahedral growth, or 3) complex internal arrangements that in part display the above characteristics, but also involve abrupt changes in crystal habit or the dominant growth mechanism. Microdiamonds characterised by internal uniformity or a patchy and diffuse distribution of luminescence centres appear to be the product of single-stage growth, but examples of homogeneity are largely subordinate to crystals possessing simple and multi-stage zonation. In general cuboids and cubo-octahedra have the least complex body structures in any suite and are essentially poor in luminescence character. Few samples of this nature contain the 'agate-style' zonation described by Bulanova and Griffin (1995) or exhibit earlier generations of diamond which have acted as nuclei for cubic growth. The majority of kimberlite 
microdiamonds (and subsequently octahedral and dodecahedroidal crystals) are comprised almost entirely of primary growth structures, with or without a seed or distinctive point of nucleation. Successive stages of growth, typically less than $20 \mu \mathrm{m}$ in width, are accompanied by marked changes in cathodoluminescence intensity that may also reflect intermittent resorption or the presence of earlier generations of diamond acting as cores or inclusions. Viewed in section, a large proportion of simply-zoned crystals demonstrate concentric growth in which minor structural anomalies disrupt the smooth, planar layering expected for ideal octahedral growth. More uncommon are examples of rough octahedral layering in which the octahedral zonation is exemplified by a characteristic 'zig-zag' structure under cathodoluminescence (Bulanova and Milledge, 1995). Complex internal structures are confined to fewer crystals, but still make up a significant portion of the cathodoluminescence patterns observed in kimberlite microdiamonds. Broadly assigned to one of the following genetic groups, the uniqueness of these images severely hampers any direct analogies between complexly-zoned stones; 1) rounded $\rightarrow$ octahedron, 2) cube $\rightarrow$ octahedron, 3) cube $\rightarrow$ rounded $\rightarrow$ octahedron, 4) cubooctahedron $\rightarrow$ octahedron, 5) cubo-octahedron $\rightarrow$ rounded $\rightarrow$ octahedron, 6) octahedron $\rightarrow$ cube, 7) polycentric. Figure 1 illustrates the level of internal variation that exists for a set of morphologically distinct microdiamonds.

\section{Nitrogen content and aggregation}

Results from FT-IR spectroscopy show that two classes of diamond, nitrogen-deficient and nitrogenbearing, are present among the microdiamonds derived from kimberlitic hosts. Contributions from both species are substantial, but the majority of microdiamonds are affiliated with substitutional nitrogen whose characteristics are dependent in part on crystal morphology. Nitrogen aggregation is notably impeded amongst cuboids and cubo-octahedra which show a strong affiliation with impurities attributed to singly-substituted and paired nitrogen defects. Most stones of this nature incorporate nitrogen purely as A-centres, however examples of mixed Ib-IaA and limited IaA-IaB aggregation are commonly dispersed throughout the sample sets. In contrast, octahedra or dodecahedroids displaying an affiliation with the Ib-IaA aggregation spectrum are rather more exceptional, and even samples accommodating nitrogen exclusively as A-defects are largely outnumbered by stones in possession of mixed A- and B-defects. The composition of nitrogen defects in type Ia microdiamonds ranges from pure IaA to $100 \% \mathrm{IaB}$, but despite the broad spread it appears most samples are poor in B-defects with very few experiencing conditions favourable for extensive A-B conversion. Likewise the range of nitrogen contents for kimberlite-derived microdiamonds is considerable, spanning from negligible or not detectable levels of nitrogen to more than 2400 atomic ppm. The distribution pattern, however, is by no means uniform, induced in part by a salient component of nitrogen-deficient and nitrogenpoor stones and the high levels of nitrogen typically acquired by stones of cubic descent. Consequently the greatest proportion of stones contain negligible or low levels of nitrogen $(<1000$ atomic ppm).

\section{Discussion}

The conditions surrounding the development of nitrogen-free diamond are as yet unknown, but the thermal history accompanying type I microdiamonds prior to their extrusion at the earth's surface can be broadly constrained by their nitrogen aggregation characteristics. Significant nitrogen aggregation in kimberlite microdiamonds, combined with the apparent sparsity of single-substitution defects, suggests an origin concurrent with kimberlite emplacement is unfeasible for most microdiamond components. Limited IaA-IaB aggregation linked with moderate nitrogen contents indicate microdiamonds form within the temperature regime, possibly over large periods of time as expected for their macrodiamond counterparts. Likewise, the non-uniform and multistage growth patterns revealed by cathodoluminescence imaging are unlikely to complement a rapid or instantaneous derivation in any source environment. Microdiamonds probably grow in multiple events or continuously, however the diversity present within the studied microdiamonds cannot be reconciled with a single environment for microdiamond genesis. Instead these microdiamonds share many features commonly reported for macrodiamonds, which would suggest a common origin. The possibility, however, of other crystals, specifically those deficient in nitrogen and demonstrating single-stage growth, developing in other sources favourable to diamond development cannot be eliminated. 


\section{References}

Bulanova, G.P. and Griffin, B.J., 1995, The origin of complex "agate" textures in octahedral diamonds from kimberlites. Extended Abstracts, Sixth International Kimberlite Conference, 74-76.

Bulanova, G.P. and Milledge, H.J., 1995, Origin and history of growth of macrodiamonds from Yakutian kimberlites. Extended Abstracts, Sixth International Kimberlite Conference, 77-79.

Haggerty S.E., 1986, Diamond genesis in a multiply-constrained model. Nature, 320, 34-38.

Trautman, R.L., Griffin, B.J., Taylor, W.R., Spetsius, Z.V., Smith, C.B. and Lee, D.C., 1997, A comparison of the microdiamonds from kimberlite and lamproite of Yakutia and Australia. Proceedings of the Sixth International Kimberlite Conference, Volume 2, Russian Geology and Geophysics, 38, 341-355.

Pattison D.R.M. and Levinson A.A., 1995, Are euhedral microdiamonds formed during ascent and decompression of kimberlite magma? Implications for use of microdiamonds in diamond grade estimation. Applied Geochemistry, 10, 725-738.
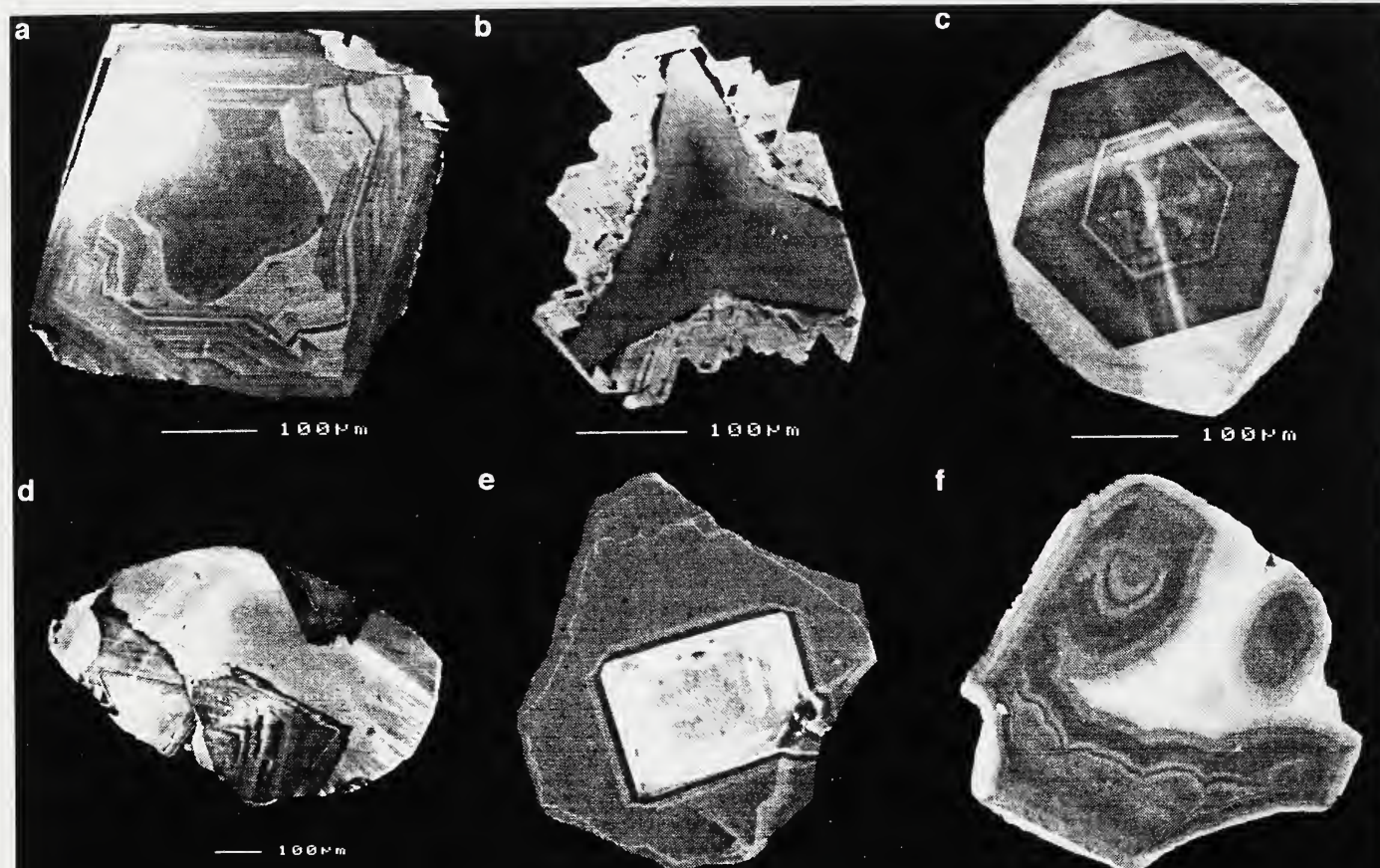

e
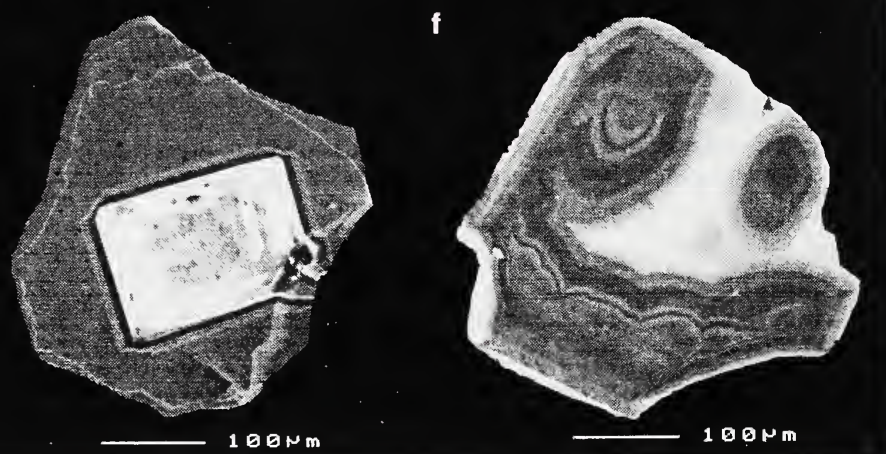

Figure 1. Growth structures of kimberlite microdiamonds under cathodoluminescence

a) Octahedron: classic cubo-octahedron $\rightarrow$ octahedron zonation (Aikhal kimberlite) b) Octahedron: dendritic/cubic $\rightarrow$ roughlayered octahedron zonation (Sytykanskaya kimberlite) c) Dodecahedroid: sectorial development during growth of a planar dodecahedron? (Sputnik kimberlite) d) Dodecahedroid: polycentric development (Yubileynaya kimberlite) e) Cuboid: octahedron $\rightarrow$ cube zonation (Yubileynaya kimberlite) f) Cuboid: 'agate' style zonation (Excalibur kimberlite) 